\title{
Some Remarks on Neutrino Oscillation and Masses
}

\author{
A. Zee* \\ Kavli Institute for Theoretical Physics \\ University of California \\ Santa Barbara, CA 93106, USA
}

September 3, 2018

\begin{abstract}
We discuss an Ansatz for the neutrino mixing matrix and speculate on the form and origin of the neutrino mass matrix.
\end{abstract}

\section{Mixing Matrix}

Thanks to heroic experimental efforts, the neutrino mixing matrix has now been determined to be 1] (see the various experimental talks at this conference)

$$
V=\left(\begin{array}{lll}
0.72-0.88 & 0.46-0.68 & <0.22 \\
0.25-0.65 & 0.27-0.73 & 0.55-0.84 \\
0.10-0.57 & 0.41-0.80 & 0.52-0.83
\end{array}\right)
$$

(The numbers given are absolute values of the matrix elements of V.) The mixing matrix $V$ relates the neutrinos current eigenstates (denoted by $\nu_{\alpha}(\alpha=e, \mu$, $\tau)$ and coupled by the $W$ bosons to the corresponding charged leptons) to the neutrino mass eigenstates (denoted by $\nu_{i}(i=1,2,3)$ ) according to

$$
\left(\begin{array}{c}
\nu_{e} \\
\nu_{\mu} \\
\nu_{\tau}
\end{array}\right)=V\left(\begin{array}{l}
\nu_{1} \\
\nu_{2} \\
\nu_{3}
\end{array}\right)
$$

We will take the neutrinos to be Majorana 2] as seems likely, so that we have in the Lagrangian the mass term

$$
\mathcal{L}=-\nu_{\alpha} M_{\alpha \beta} C \nu_{\beta}+\text { h.c. }
$$

*zee@kitp.ucsb.edu 
where $C$ denotes the charge conjugation matrix. Thus, the neutrino mass matrix $M$ is symmetric. For the sake of simplicity we will assume $C P$ conservation so that $M$ is real. Indeed, we have already written all the entries as real in (1).

With this simplification, $M$ is diagonalized by an orthogonal transformation

$$
V^{T} M V=\left(\begin{array}{lll}
m_{1} & 0 & 0 \\
0 & m_{2} & 0 \\
0 & 0 & m_{3}
\end{array}\right)
$$

Clearly, we are free to multiply $V$ on the right by some diagonal matrix whose diagonal entries are equal to \pm 1 . This merely multiplies each of the columns in $V$ by an arbitrary sign. Various possible phases have been discussed in detail in the literature. 3

We could suppose either that the entries in $V$ represent a bunch of meaningless numbers or that they point to some deeper structure or symmetry. In the latter spirit, let us make a guess of what $V$ might be.

Since $V_{e 3}$ appears to be small, let us boldly set it to 0 . Next, since $1 / \sqrt{2} \sim$ 0.707 we will guess that $V_{\mu 3}=(0.55-0.84)=1 / \sqrt{2}$. Finally, since $1 / \sqrt{3} \sim 0.577$ we will set $V_{e 2}=(0.46-0.68)=1 / \sqrt{3}$. In other words, we propose that we know the upper triangular entries of the matrix $V$ :

$$
V=\left(\begin{array}{ccc}
X & \frac{1}{\sqrt{3}} & 0 \\
X & X & \frac{1}{\sqrt{2}} \\
X & X & X
\end{array}\right)
$$

where $X$ denotes an unknown quantity.

Remarkably, this essentially fixes the mixing matrix $V$. Once we take the last column to be proportional to $(0,1,-1)$, orthogonality and our "knowledge" that $V_{e 2}$ is $1 / \sqrt{3}$ immediately fix the second column to be proportional $(1,1,1)$ and hence the first column to be proportional to $(-2,1,1)$. X. G. He and I therefore arrived at the Ansatz or guess 4

$$
V=\left(\begin{array}{rrr}
-\frac{2}{\sqrt{6}} & \frac{1}{\sqrt{3}} & 0 \\
\frac{1}{\sqrt{6}} & \frac{1}{\sqrt{3}} & \frac{1}{\sqrt{2}} \\
\frac{1}{\sqrt{6}} & \frac{1}{\sqrt{3}} & -\frac{1}{\sqrt{2}}
\end{array}\right) \text {. }
$$

This mixing matrix (but curiously, with the first and second column interchanged) was first suggested by Wolfenstein more than 20 years ago [5]. Later it was proposed by Harrison, Perkins and Scott [6], and subsequently studied extensively by them and by Xing $[$.

The mixing matrix $V$ may be factorized as $V=V_{23} V_{12}$ where

$$
V_{23}=\left(\begin{array}{ccc}
1 & 0 & 0 \\
0 & \frac{1}{\sqrt{2}} & \frac{1}{\sqrt{2}} \\
0 & \frac{1}{\sqrt{2}} & -\frac{1}{\sqrt{2}}
\end{array}\right)
$$


and

$$
V_{12}=\left(\begin{array}{rrr}
-\sqrt{\frac{2}{3}} & \frac{1}{\sqrt{3}} & 0 \\
\frac{1}{\sqrt{3}} & \sqrt{\frac{2}{3}} & 0 \\
0 & 0 & 1
\end{array}\right) .
$$

In other words, if we follow Wolfenstein and define $\nu_{x} \equiv\left(\nu_{\mu}+\nu_{\tau}\right) / \sqrt{2}$ and $\nu_{y} \equiv\left(-\nu_{\mu}+\nu_{\tau}\right) / \sqrt{2}$ we find that the mass eigenstates are given by

$$
\begin{gathered}
\nu_{1}=-\sqrt{\frac{2}{3}} \nu_{e}+\frac{1}{\sqrt{3}} \nu_{x}, \\
\nu_{2}=\frac{1}{\sqrt{3}} \nu_{e}+\sqrt{\frac{2}{3}} \nu_{x},
\end{gathered}
$$

and

$$
\nu_{3}=\nu_{y}
$$

I find this matrix $V$ rather attractive, but how could we obtain such an "elegantly simple" mixing matrix?

Recently, Harrison, Perkins and Scott $[8]$ proposed a discrete symmetry group $\mathcal{D}$ and managed to obtain $V$. Unfortunately, as emphasized by Low and Volkas 9 , they have to allow the left handed neutrinos and the left handed charged leptons, which of course belong to the same doublet under the standard $S U(2) \otimes U(1)$, to transform differently. Thus the low energy symmetry group of the electroweak interaction proposed by Harrison et al could not have the form $S U(2) \otimes U(1) \otimes \mathcal{D}$. Assuming that it indeed has this form and assuming only one Higgs doublet, Low and Volkas went on and proved a no-go theorem showing that no choice of $\mathcal{D}$ would lead to $V$.

\section{Mass Matrix}

Neutrino oscillation [10] experiments can only determine the absolute value of the mass squared differences $\Delta m_{i j}^{2} \equiv m_{i}^{2}-m_{j}^{2}$. At the $99.3 \%$ confidence level $\Delta m_{i j}^{2}$ are determined by

$$
1.5 \times 10^{-3} \mathrm{eV}^{2} \leq\left|\Delta m_{32}^{2}\right| \leq 5.0 \times 10^{-3} \mathrm{eV}^{2},
$$

and

$$
2.2 \times 10^{-5} \mathrm{eV}^{2} \leq\left|\Delta m_{21}^{2}\right| \leq 2.0 \times 10^{-4} \mathrm{eV}^{2},
$$

with the best fit values given by

$$
\left|\Delta m_{32}^{2}\right|=3.0 \times 10^{-3} \mathrm{eV}^{2}
$$

and

$$
\left|\Delta m_{21}^{2}\right|=7.0 \times 10^{-5} \mathrm{eV}^{2}
$$


Thus, we could have either the so-called normal hierarchy in which $\left|m_{3}\right|>$ $\left|m_{2}\right| \sim\left|m_{1}\right|$ or the inverted hierarchy $\left|m_{3}\right|<\left|m_{2}\right| \sim\left|m_{1}\right|$. At present, we have no understanding of the neutrino masses just as we have no understanding of the charged lepton and quark masses.

In general, when we diagonalize a matrix $M$ as in (4) we expect the eigenvalues $m_{i}$ and the matrix $V$ to depend on the matrix elements $M_{\alpha \beta}$. Only a certain class of matrices would have the property (which Low and Volkas called "form-diagonalizable") such that $V$ comes out as a matrix of pure numbers as in (5). At first sight, it seems a bit odd that form-diagonalizable matrices exist, but a moment's thought indicates that they could be constructed as follows: given three orthonormal column vectors $\vec{v}^{(i)}=\left\{v_{\alpha}^{(i)}\right\}$ whose components are pure numbers, then $M=\sum_{i=1}^{3} m_{i} \vec{v}^{(i)}\left(\vec{v}^{(i)}\right)^{T}$ is form diagonalizable for arbitrary $m_{i}$. Thus, if we believe in (5) then the neutrino mass matrix is given by

$$
M=\frac{m_{1}}{6}\left(\begin{array}{lll}
4 & -2 & -2 \\
-2 & 1 & 1 \\
-2 & 1 & 1
\end{array}\right)+\frac{m_{2}}{3}\left(\begin{array}{lll}
1 & 1 & 1 \\
1 & 1 & 1 \\
1 & 1 & 1
\end{array}\right)+\frac{m_{3}}{2}\left(\begin{array}{lll}
0 & 0 & 0 \\
0 & 1 & -1 \\
0 & -1 & 1
\end{array}\right)
$$

The three column vectors contained in $V$ are the eigenvectors of the matrix

$$
M_{0}=a\left(\begin{array}{rrr}
2 & 0 & 0 \\
0 & -1 & 3 \\
0 & 3 & -1
\end{array}\right)
$$

with eigenvalues $m_{1}=m_{2}=2 a$, and $m_{3}=-4 a$. (The parameter $a$ merely sets the overall scale.) Thus, $\Delta m_{21}^{2}=0$ and this pattern reproduces the data $\left|\Delta m_{21}^{2}\right| /\left|\Delta m_{32}^{2}\right| \ll 1$ to first approximation. Because of the degeneracy in the eigenvalue spectrum, $V$ is not uniquely determined. We can always replace $V$ by $V W$ where

$$
W=\left(\begin{array}{cc}
R & 0 \\
0 & 1
\end{array}\right)
$$

with $R$ a $2 \times 2$ rotation matrix. To determine $V$, and at the same time to split the degeneracy between $m_{1}$ and $m_{2}$, we perturb $M_{0}$ to $M=M_{0}+\delta M_{T}$, where

$$
\delta M_{T}=\varepsilon a\left(\begin{array}{lll}
0 & 1 & 1 \\
1 & 0 & 1 \\
1 & 1 & 0
\end{array}\right) .
$$

We have the mass eigenvalues $m_{1}=2 a(1-\varepsilon / 2), m_{2}=2 a(1+\varepsilon)$, and $m_{3}=$ $-4 a(1+\varepsilon / 4)$. Thus, we can determine to the lowest order $\varepsilon=\Delta m_{21}^{2} / \Delta m_{32}^{2}$. The overall scale of the mass matrix $a$ is given by $a^{2}=\Delta m_{32}^{2} / 12$.

Other perturbations can also lead to the same mixing matrix $V$ while splitting the degeneracy $\Delta m_{21}^{2}=0$. An interesting example is the "democratic" form 


$$
\delta M_{D}=\varepsilon a\left(\begin{array}{lll}
1 & 1 & 1 \\
1 & 1 & 1 \\
1 & 1 & 1
\end{array}\right) .
$$

The matrix $\delta M_{D}$ is evidently a projection matrix that projects the first and third columns in $V$ to zero. Thus, the eigenvalues are given by $m_{1}=2 a, m_{2}=$ $2 a(1+3 \varepsilon / 2)$, and $m_{3}=-4 a$, where to the lowest order $\varepsilon=\Delta m_{21}^{2} / \Delta m_{32}^{2}$ and $a^{2}=\Delta m_{32}^{2} / 12$. We note that this mass matrix is not traceless.

We mention that there is a whole class of models we can propose. Generalize $M_{0}$ to

$$
\tilde{M}_{0}=a\left(\begin{array}{ccc}
2 & 0 & 0 \\
0 & 1-y & 1+y \\
0 & 1+y & 1-y
\end{array}\right),
$$

with the case mentioned earlier corresponding to $y=2$. Thus in general we propose

$$
M=\tilde{M}_{0}+\delta M,
$$

with $\delta M$ being $\delta M_{T}$ or $\delta M_{D}$. They lead to the same mixing matrix $V$, with the eigenvalues $m_{i}$ given by $(2 a(1-\varepsilon / 2), 2 a(1+\varepsilon),-2 a(y+\varepsilon / 2))$ and $(2 a, 2 a(1+$ $3 \varepsilon / 2),-2 a y)$, respectively.

Note that the most general mass matrix which produces the mixing matrix $\mathrm{V}$ can be expressed as linear combinations of the three matrices of the forms given by $M_{0}, \delta M_{T}$ and $\delta M_{D}$. Once we committed to a specific form for $M$, the three parameters specifying the linear combination merely parametrize the three neutrino masses $m_{1,2,3}$. Obviously for any given mixing matrix, the mass matrix can be specified by mass eigenvalues.

With 2 experimental numbers for the 3 masses $m_{1,2,3}$, we have to make another wild guess in order to determine $M$. Since $M=M_{0}+\delta M_{T}$ gives a reasonable fit to the data and since it is traceless, one may be tempted to conjecture that this property provides a clue to the origin of the neutrino mass matrix. In a recent paper [1], X. G. He and I gave a phenomenological analysis of the data imposing the condition[12 $\operatorname{Tr} M=0$ without speculating on its theoretical origin.

If there is no $C P$ violation in the neutrino mass matrix $M$, the mass matrix can always be made real and it can be diagonalized by an orthogonal transformation. In this case the traceless condition $\operatorname{Tr} M=0$ is equivalent to the "zero sum" condition

$$
m_{1}+m_{2}+m_{3}=0 .
$$

(But if $C P$ is not conserved, the "zero sum" and traceless conditions are different. One needs to be careful about the phase definitions [3].) We note that 
the traceless condition holds if $M=[A, B]$, that is, the mass matrix can be expressed as a commutator of two matrices $A$ and $B$.

As remarked earlier, we are free to choose the signs of the column vectors in the mixing matrix and to make chiral rotations on the neutrino fields to change the relative signs of the mass eigenvalues. Without information on the relative signs of the eigen-masses, the column vectors can only be determined up to $\pm i$. This can be expressed by multiplying a diagonal phase matrix $P=$ $\operatorname{Diag}\left(e^{i \sigma}, e^{i \rho}, 1\right)$ to the right of $V$. With $C P$ invariance, $\sigma$ and $\rho$ can take the values of zero or $\pm \pi / 2$. Neutrinoless double beta decays will provide some crucial information on these phases.

Combining the zero mass condition with the experimental data on the differences of mass squared we find that the mass eigenvalues exhibit two types of hierarchies,

$$
\begin{aligned}
& \text { (I) } m_{3} \approx-2 m_{1} \approx-2 m_{2} \approx 0.064 \mathrm{eV} \\
& \text { (II) } m_{1} \approx-m_{2} \approx 0.054 \mathrm{eV}, \text { and } m_{3} \approx 0.00064 \mathrm{eV} .
\end{aligned}
$$

The sign of $\Delta m_{32}^{2}$ decides which mass hierarchy the solutions belong to. Note that the "natural" sign $\Delta m_{32}^{2}>0$ corresponds to scenario $(I)$, in which the

masses are of the same order of magnitude, in contrast to scenario $(I I)$, in which $m_{3}$ is two order of magnitude smaller than $m_{1}$ and $m_{2}$. We would like to suggest that $(I)$ is favored over $(I I)$.

Our purpose here is evidently not to give a detailed fit to the data, but to suggest some relatively simple and appealing mass matrices. The appearance of simple integers in the mixing and mass matrices we proposed is perhaps intriguing and provides a glimmer of a hope that they may be obtained by group theoretic considerations. To provide a theoretical origin of the mass matrix $M$ presents an interesting challenge.

\section{Theory}

Theoretically, pitifully little is known about neutrino masses and mixing. The only firm theoretical statement is that since the standard model is correct at low energies, neutrino masses have to come from the following dimension 5 operator in the Lagrangian, 13, 14, 15.

$$
\mathcal{L}=\frac{1}{\mathcal{M}}\left(\varepsilon_{i j} \psi_{i} \varphi_{j}\right) C\left(\varepsilon_{k l} \psi_{k}^{\prime} \varphi_{l}^{\prime}\right)
$$

Here $\psi$ and $\psi^{\prime}$ denote left handed lepton doublets and $\varphi$ and $\varphi^{\prime}$ Higgs doublets, and $i, j, k, l$ denote $S U(2)$ indices. The unknown mass parameter $\mathcal{M}$ sets the mass scale of the new physics responsible for generating the neutrino masses.

Life is full of bifurcating choices: so too the neutrino mass model builder is immediately faced with the choice of introducing right handed neutrinos or not. A neutrino could have either a Dirac mass or a Majorana mass. In the first alternative, one needs to introduce right handed neutrino fields and the question 
immediately arises on why the neutrino Dirac masses are so small compared to the charged lepton masses in the theory. This question was answered elegantly by the see-saw mechanism, in which the right handed neutrino fields are given large Majorana masses. But if we are willing to introduce Majorana masses for the right handed neutrino fields, perhaps we should consider dispensing with right handed neutrino fields altogether and simply try to generate Majorana masses for the existing left handed neutrino fields. We shall try to generate this Majorana masses through quantum mechanical effect. This has the added advantage of having naturally small neutrino mass and the new physics at a potentially experimentally accessible scale.

Since in the standard model, the left handed neutrino fields belong to doublets $\psi_{a L}$ (with $a$ a family index) we cannot simply put in Majorana mass terms. The general philosophy followed in Ref. [16, 17, 18] is that we should feel freer to alter the scalar field sector than other sectors since the scalar field sector is the least established one in the standard model. Out of the doublets we can form the Lorentz scalar $\left(\psi_{a L}^{i} C \psi_{b L}^{j}\right)$ (where $i, j$ denote electroweak $S U(2)$ indices and $C$ the charge conjugation matrix): this can be either a triplet or a singlet under $S U(2)$. If we couple a triplet field to this lepton bilinear, then when the neutral component of the triplet field acquires a vacuum expectation value, the neutrinos immediately acquire Majorana masses. We considered this model unattractive: not only does it lack predictive power, but the rather accurately studied ratio of $W$ and $Z$ boson masses puts a stringent bound on any triplet Higgs. In addition, there is no natural way to explain the smallness required of this vacuum expectation value.

We thus chose the alternative of coupling to an $S U(2)$ singlet (charged) field $h^{+}$via the term $f^{a b}\left(\psi_{a L}^{i} C \psi_{b L}^{j}\right) \varepsilon_{i j} h^{+}$. An interesting point is that due to Fermi statistics the coupling $f^{a b}$ must be anti-symmetric in $a$ and $b$. We are forced to couple leptons in one family to leptons in another one. Thus, the term above contains $f^{e \mu}\left(\nu_{e} C \mu^{-}-e^{-} C \nu_{\mu}\right) h^{+}$, for instance. The term $f^{a b}\left(\psi_{a L}^{i} C \psi_{b L}^{j}\right) \varepsilon_{i j} h^{+}$ in itself does not violate lepton number $L$ since we can always assign $L=$ -2 to $h^{+}$. But we note that we can also couple $h^{+}$to the Higgs doublets via $M_{\alpha \beta} \phi_{\alpha} \phi_{\beta} h^{+}$if there are more than one Higgs doublet. By Bose statistics, the coupling matrix $M_{\alpha \beta}$ is antisymmetric and thus we need to have at least two Higgs doublets. We do not regard the necessity of more than one Higgs doublet as unattractive. Indeed, theorists have always been motivated by one reason or another to introduce additional Higgs doublets, and surely in the debris of breaking down from some high mass scale physics there would be numerous scalar fields. If the doublets are required to have zero lepton number by their respective Yukawa couplings, the term $M_{\alpha \beta} \phi_{\alpha} \phi_{\beta} h^{+}$violates lepton number by two units, just right for generating neutrino Majorana masses.

From general principles we know that neutrino Majorana masses must be generated and that they must come out as finite, that is, calculable in terms of the parameters of the theory. Indeed, it is easy to see that calculable neutrino Majorana masses are generated by quantum fluctuations in one loop 16.

There has been a considerable literature 19] devoted to studying the various implications of this model, known as the Zee model. Soon after this model was 
proposed, Wolfenstein 20] suggested an interesting simplification by imposing a discrete symmetry so that one of the two minimally necessary Higgs doublets does not couple to leptons. This simplified Zee model, or the Zee-Wolfenstein model, is now ruled out 21. The original Zee model, however, continues to be phenomenologically viable 22,23 . There are also several possible variants [24] of this model. It would be interesting to see if the mixing and mass matrices discussed in the first half of this paper could possibly emerge from this class of models.

\section{Acknowledgments}

Part of the material discussed in this paper is based on work done with $\mathrm{X}$. $\mathrm{G}$. He. I thank R. Volkas, C. N. Leung, and S. Tovey for inviting me to speak at this conference. I would also like to thank J. Nieves for computer help. This work was supported in part by the National Science Foundation under grant number PHY 99-07949.

\section{References}

[1] Concha Gonzalez-Garcia, http://www.dpf2003.org/xx/neutrino/concha.pdf J.N. Bachall, M.C. Gonzalez-Garcia and C. Pena-Garay, arXiv: hep-ph/0212147

[2] For a review, see for example, B. Kayser, hep-ph/0211134

[3] J. F. Nieves and P. B. Pal, Phys. Rev. D36, 315(1987); S. Bilenky and S. Pascoli and S. Petcov, Phys. Rev. D64, 053010(2001).

[4] X. G. He and A. Zee, hep-ph/0301092.

[5] L. Wolfenstein, Phys. Rev. D18, 958(1978)

[6] P.F. Harrison, D. H. Perkins and W.G. Scott, Phys. Lett. B458, 79(1999); W.G. Scott, Nucl. Phys. Proc. Suppl. 85, 177(2000) [arXiv: hep-ph/9909431; P. F. Harrison and W.G. Scott, Phys. Lett. B535, 163(2002); P.F. Harrison and W.G. Scott, arXiv: hep-ph/0302025

[7] Z.-Z. Xing, Phys. Lett. B533, 85(2002) [arXiv: hep-ph/0204049.

[8] P. F. Harrison, D.H. Perkins and W.G. Scott, Phys. Lett. B530, 167(2002) [arXiv: hep-ph/0202074

[9] C. I. Low and R. R. Volkas, hep-ph/0305243

[10] B. Pontecorvo, Sov. Phys. JETP 6, 429(1957); 7, 172(1958); Z. Maki, M. Nakagawa and S. Sakata, Prog. Theor. Phys. 28, 870(1962); B. Lee, S. Pakvasa, R. Shrock and H. Sugawara, Phys. Rev. Lett. 38, 937(1977); B. Lee and R. Shrock, Phys. rev. D16, 1444(1977). 
[11] X. G. He and A. Zee, hep-ph/0302201.

[12] This "zero sum" Ansatz was also proposed earlier by D. Black, A. Fariborz, S. Nasri and J. Schechter, Phys. rev. D62, 073015(2000). Their theoretical motivation and the values of the masses they obtained are however rather different.

[13] S. Weinberg, Phys. Rev. Lett. 43, 1566(1979).

[14] F. Wilczek and A. Zee, Phys. Rev. Lett. 43, 1571(1979).

[15] A. Weldon and A. Zee, Nucl. Phys. B173, 269 (1980).

[16] A. Zee, Phys. Lett. 93B, 389(1980).

[17] A. Zee, Phys. Lett. 161B, 141(1985).

[18] A. Zee, Nucl. Phys. B264 99 (1986).

[19] Y. Smirnov and Zhijian Tao, Nucl. Phys. B426, 415(1994); Y. Smirnov and M. Tanimoto, Phys. Rev. D55, 1665(1997); C. Jarlskog et al., Phys. Lett. B449, 240(1999); P. Frampton and S. Glashow, Phys. Lett. B461, 95(1999); Y. Koide, Phys. Rev. D64, 077301(2001); P. Frampton, M. Oh and T. Yoshikawa, Phys. Rev. D65, 073014(2002); A. Joshipura and S. Rindani, Phys. Lett. B464, 239(1999); K.-M. Cheung and O. Kong, Phys. Rev. D16, 113012(2000); K. Baliji, W. Grimus and T. Schwetz, Phys. Lett. B508, 301(2001); E. Mitsuda and K. Sasaki, Phys. Lett. B516, 47(2001); A. Ghosal, Y. Koide and H. Fusaoka, Phys. Rev. D64, 053012(2001); D. Dicus, H.-J. He and J. Ng, Phys. Rev. Lett. 87, 111803(2001); B. Brahmachari and S. Choubey, Phys. Lett. B531, 99(2002); T. Kitabayashi and M. Yasue, Int.J. Mod. Phys. A17, 2519(2002).

[20] L. Wolfenstein, Nucl. Phys. B175, 93(1980).

[21] See for example P. H. Frampton, M. C. Oh, T. Yoshikawa, Phys.Rev. D65 (2002) 073014; hep-ph/0110300

[22] K.Hasegawa, C. S. Lim, K. Ogure, hep-ph/0303252

[23] X. G. He, to be published.

[24] For a review and references to the literature, see D. Chang and A. Zee, Phys.Rev. D61, 071303 (2000), hep-ph/9912380 